\title{
Modelling DTPA decorporation of Am in rats
}

\author{
Manuel Kastl ${ }^{1,2^{*}}$, Olivier Grémy $^{3}$, Laurent Miccoli ${ }^{3}$, Stéphanie Lamart ${ }^{3}$, Weibo $\mathrm{Li}^{1}$, and \\ Augusto Giussani ${ }^{4}$ \\ ${ }^{1}$ Helmholtz Zentrum München, German Research Center for Environmental Health, 85764 \\ Neuherberg, Germany \\ ${ }^{2}$ Technische Universität München, Fakultät für Physik, 85748 Garching, Germany \\ ${ }^{3}$ Laboratoire de Radiotoxicologie CEA/LRT, Université de Paris-Saclay, Bruyères-le-Châtel, France \\ ${ }^{4} \mathrm{BfS}$ - Federal Office for Radiation Protection - Department of Radiation and Health, 85764 \\ Oberschleißheim, Germany
}

Decorporation therapy with DTPA (diethylene triamine pentaacetic acid) is recommended as a standard procedure after accidental intake of transuranics such as plutonium/americium $(\mathrm{Pu} / \mathrm{Am})$. DTPA is able to chelate the incorporated $\mathrm{Pu} / \mathrm{Am}$ and eliminate them rapidly from the body, mainly by enhancing their urinary excretion rate. In general $\mathrm{Ca} / \mathrm{Zn}-\mathrm{DTPA}$ is administered following empirical protocols. Since the chelation process perturbs the regular biokinetics of $\mathrm{Pu} / \mathrm{Am}$, standard biokinetic models are inadequate for interpreting monitoring data and estimating dose to the patient. Moreover, optimization of the treatment strategy and prospective assessments of the therapy success in terms of averted dose cannot be evaluated by using the current models.

In the recent years a European collaboration within the frame of EURADOS (European Radiation Dosimetry Network) has been initiated to develop a compartmental model approach to this issue. The basic idea of the adopted approach is to consider the biokinetics of $\mathrm{Pu} / \mathrm{Am}$ and of the injected DTPA separately and to couple them by a suitable mathematical description of the chelation mechanism as a second-order process $[1,2]$. Except the proposal presented by Konzen and Brey [3], which is based on the EURADOS approach, all other attempts existing in the literature [4-9] are empirical and developed mainly for the interpretation of one or a limited number of specific incorporation cases.

In this work the available experimental data from controlled animal studies with Am recently published by Grémy et al. [10] was used to define the biological sites of chelation and the chelation rate constants. Rats were given DTPA either prior to or after Am intravenous injection. In these studies all boundary conditions are precisely known, differently from the case of accidental intakes in humans. The chelation rate constants and the identification of the chelation sites are thus the only unknown variables of the system.

In a first step, chelation was assumed to occur only in extracellular fluids, with two different rate constants for chelation in blood and chelation in other fluids (lymph, interstitial fluids). Model predictions reproduce the accumulation of activity in urine until time of sample collection, corresponding to a $24 \mathrm{~h}$ period in the studies of Grémy et al. Predictions were compared with the experimental $24 \mathrm{~h}$ urinary excretions (Fig. 1). While there is a good agreement between prediction and data when DTPA has been administered shortly after contamination (top left panel), the agreement for the other experiments is less satisfactory. Moreover, increased faecal excretion and decorporation from liver and

*Corresponding author: manuel.kastl@helmholtz-muenchen.de 
skeleton cannot be reproduced under these simple assumptions. For this reason, further simulations are planned, considering in addition intracellular chelation.

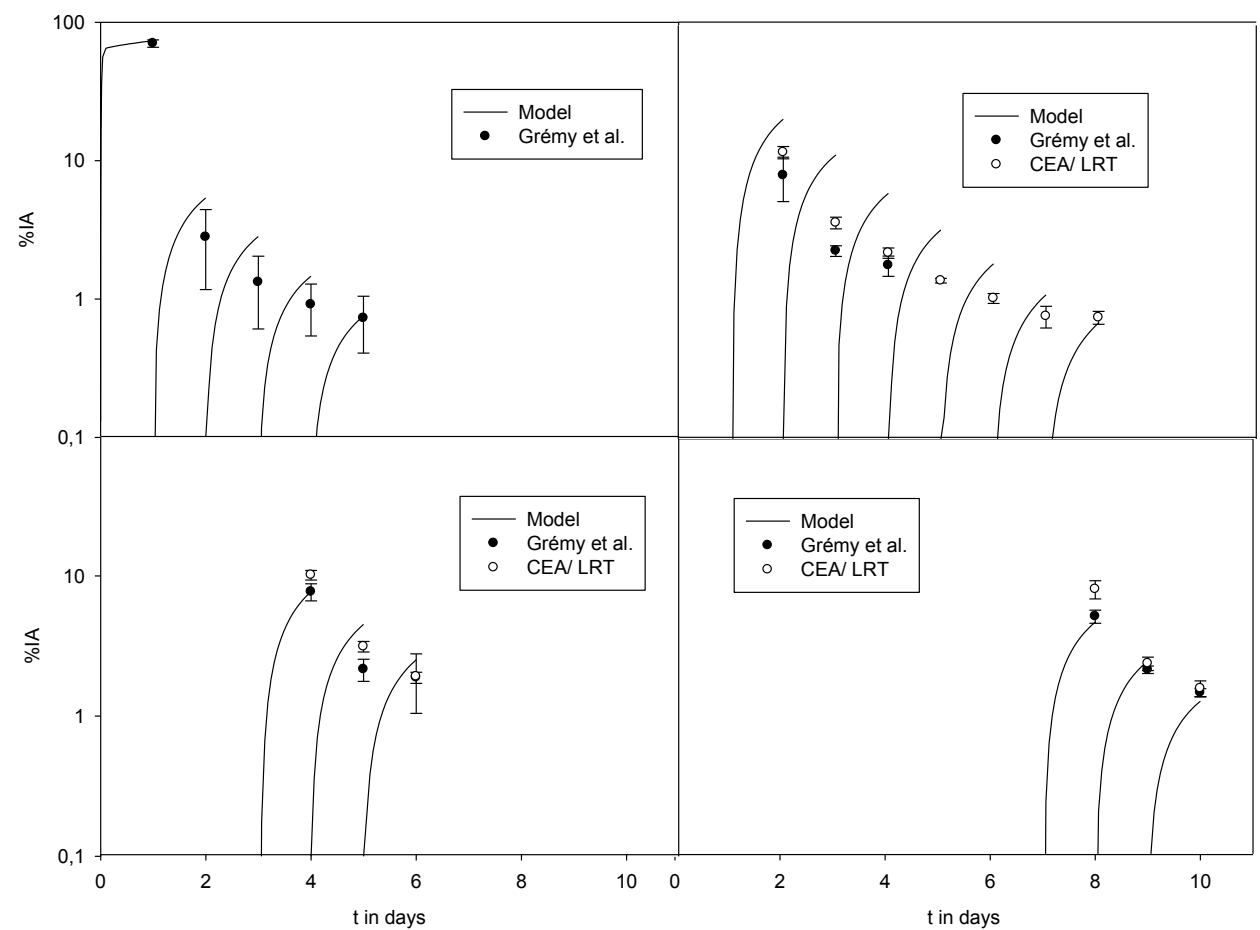

Figure 1. Comparison of measured $24 \mathrm{~h}$ urine excretion with the corresponding model predictions for single DTPA injection at different times after contamination: 3 minutes ( top left), one day (top right), three days (bottom left) and seven days (bottom right). Values are expressed as the percentage of the initial Injected Activity (mean \pm sd (\% IA)). "Grémy et al." are data presented in [10]; "CEA/LRT" are unpublished data from the same laboratory.

\section{References}

1. B. Breustedt, E. Blanchardon, P. Berard, et al., Radiat. Prot. Dosim. 1341 (2009)

2. M. Kastl, A. Giussani, E. Blanchardon, B. Breustedt, P. Fritsch, C. Hoeschen, M.A. Lopez, Int. J. Radiat. Biol. 90 (2014)

3. K. Konzen and R. Brey, Health Phys. 108 (2015)

4. R.R. Bailey, K.F. Eckerman, L.W. Townsend, Radiat. Prot. Dosim. 105 (2003)

5. P. Fritsch, L. Grappin, A.M. Guillermin, R. Fottorino, M. Ruffin, A. Mièle, Radiat. Prot. Dosim. 127 (2007)

6. R.M. Hall, G.A. Poda, R.R. Fleming, J.A. Smith, Health Phys. 34 (1978)

7. A.C. James, L.B. Sasser, D.B. Stuit, S.E. Glover, E.H. Carbaugh, Radiat. Prot. Dosim. 127 (2007)

8. J.J. Jech, B.V. Andersen, K.R. Heid, Health Phys. 22 (1972)

9. T.R. LaBone, Internal Radiation Dosimetry (Medical Physics Publishing, 1994)

10. O. Grémy, D. Laurent, S. Coudert, N.M. Griffiths, L. Miccoli, Rad. Res. 185 (2016) 